\title{
Introduction to the Smart Service Systems: Analytics, Artificial Intelligence and Cognitive Applications
}

\author{
Haluk Demirkan \\ U. of Washington-Tacoma \\ haluk@uw.edu
}

\author{
James C. Spohrer \\ IBM Almaden Research Center \\ spohrer@us.ibm.com
}

\author{
Ralph Badinelli \\ Virginia Tech \\ ralphb@vt.edu
}

Economic and societal well-being depend on innovations that help people use big data more intelligently. Human-centered smart service systems for business and society can be characterized by: (1) the types of offerings to their customers and/or citizens, (2) the types of jobs or roles for people within them, and (3) the types of returns they offer investors interested in growth and development, through improved use of technology, talent, or organizational and governance forms, which create (dis)incentives that (re)shape behaviors. An important trend in smart service systems is the increasing availability of cognitive assistants (e.g., Siri, Watson, Jibo, Echo, Cortana, Bixby, Einstein, and Now) to boost productivity and creativity of all the people inside them.

Innovators of smart service systems, including entrepreneurs, managers, and policymakers seek to improve quality-of-service for customers, quality-of-life for citizens, and/or quality-of-returns for investors.

Smart service systems are ones that continuously improve (e.g., productivity, quality, compliance, sustainability, etc.) and co-evolve with all sectors (e.g., government, healthcare, education, finance, retail and hospitality, communication, energy, utilities, transportation, etc.). Regional service systems include nations, states, cities, universities, and hospitals. Global service systems include multi-national businesses, professional associations, and NGOs. Natural or human-made disasters, technology failures, criminal activities, political collapse can disrupt or negatively impact quality-oflife for people living and working in service systems.

Using big data analytics and cognitive systems to improve decision-making service providers try to compete for the hearts, minds, and wallets of collaborators by (1) improving existing offerings, (2) innovating new types of offerings, (3) evolving their portfolio of offerings, and, (4) changing their relationships to others in the ecosystem in ways stakeholders perceive as more positive, sustainable, fair, or responsible.

The goal of this mini track is to explore the challenges, issues and opportunities related to innovation of smart service systems that enable value co-creation with analytics, cognitive and human systems. This is the fifth time that we are offering this minitrack since 2015. The three papers accepted for the minitrack investigate these issues in different ways.
The first paper, titled "Constraint programming for flexible Service Function Chaining deployment” by Tong Liu, Franco Callegati, Walter Cerroni, Chiara Contoli, Maurizio Gabbrielli and Saverio Giallorenzo propose the language to formalize the request of a given Service Function Chain (SFC) to the network to address SFC design problem. In their model, the request is built upon the intent-based approach, with a syntax that focuses on asking the user "what" she needs and not "how" it should be implemented, in a simple and high level language. Then, they define a formal model describing network architectures and Virtual Network Functions properties that is then used to solve the SFC design problem by means of Constraint Programming, a programming paradigm which is often used in Artificial Intelligence applications.

"Emoty: An Emotionally Sensitive Conversational Agent for People with Neurodevelopmental Disorders" by Fabio Catania, Nicola Di Nardo, Franca Garzotto and Daniele Occhiuto, aims at exploiting the advances in conversational technology to support people with Neurodevelopmental Disorder (NDD). NDD is a group of conditions that are characterized by severe deficits in the cognitive, emotional and motor areas and produce severe impairments in communication and social functioning. This paper presents the design, technology and exploratory evaluation of Emoty, a spoken Conversational Agent created specifically for individuals with NDD. The goal of Emoty is to help these persons enhancing communication abilities related to emotional recognition and expression, which are fundamental in any form of human relationship.

In the last paper titled "Classifying Smart Personal Assistants: An Empirical Cluster Analysis” by Robin Knote, Andreas Janson, Matthias Söllner and Jan Marco Leimeister, introduce a classification system for SPAs. Based on a systematic literature review, a cluster analysis reveals five smart personal assistants archetypes: Adaptive Voice (Vision) Assistants, Chatbased Assistants, Embodied Virtual Assistants, Ubiquitous Assistants, and Natural Conversation Assistants.

We hope you enjoy the papers and their presentation at the conference. We thank the authors for submitting their work to make this another engaging minitrack. We also thank the reviewers for their valuable feedback. 